\title{
Association between NRGN gene polymorphism and resting-state hippocampal functional connectivity in schizophrenia
}

Yifan Zhang ${ }^{1}$, Xiaohong Gong ${ }^{2}$, Zhiyang Yin ${ }^{1}$, Lingling Cui ${ }^{3}$, Jian Yang ${ }^{3}$, Pengshuo Wang ${ }^{1}$, Yifang Zhou ${ }^{1,4}$, Xiaowei Jiang ${ }^{1,3}$, Shengnan $W^{3} i^{3}$, Fei Wang ${ }^{1,3,5^{*}}$ and Yanqing Tang ${ }^{1,4^{*}}$ (i)

\begin{abstract}
Background: Based on genome-wide association studies, a single-nucleotide polymorphism in the NRGN gene (rs12807809) is considered associated with schizophrenia (SZ). Moreover, hippocampal dysfunction is associated with rs12807809. In addition, converging evidence suggests that hippocampal dysfunction is involved in SZ pathophysiology. However, the association among rs12807809, hippocampal dysfunction and SZ pathophysiology is unknown. Therefore, this study investigated the association between rs12807809 and hippocampal functional connectivity at rest in SZ.

Methods: In total, 158 participants were studied, including a C-carrier group carrying the non-risk C allele (29 SZ patients and 46 healthy controls) and a TT homozygous group carrying the risk T allele (30 SZ patients and 53 healthy controls). All participants were scanned using resting-state functional magnetic resonance imaging. Hippocampal functional connectivity was computed and compared among the 4 groups.

Results: Significant main effects of diagnosis were observed in the functional connectivity between the hippocampus and bilateral fusiform gyrus, bilateral lingual gyrus, left inferior temporal gyrus, left caudate nucleus, bilateral thalamus and bilateral anterior cingulate gyri. In contrast, no significant main effect of genotype was found. In addition, a significant genotype by diagnosis interaction in the functional connectivity between the hippocampus and left anterior cingulate gyrus, as well as bilateral middle cingulate gyri, was observed, with $\Pi$ homozygotes with SZ showing less functional connectivity than C-carriers with SZ and healthy control TT homozygotes.

Conclusions: These findings are the first to suggest an association between rs12807809 and abnormal Papez circuit function in patients with SZ. This study also implicates NRGN variation and abnormal Papez circuit function in SZ pathophysiology.
\end{abstract}

Keywords: Schizophrenia, NRGN, Hippocampus, fMRI, Functional connectivity

\footnotetext{
* Correspondence: fei.wang@cmu.edu.cn; tangyanqing@cmu.edu.cn

'Department of Psychiatry, The First Affiliated Hospital of China Medical

University, Shenyang, Liaoning 110001, People's Republic of China

Full list of author information is available at the end of the article
}

(c) The Author(s). 2019 Open Access This article is distributed under the terms of the Creative Commons Attribution 4.0 International License (http://creativecommons.org/licenses/by/4.0/), which permits unrestricted use, distribution, and reproduction in any medium, provided you give appropriate credit to the original author(s) and the source, provide a link to the Creative Commons license, and indicate if changes were made. The Creative Commons Public Domain Dedication waiver (http://creativecommons.org/publicdomain/zero/1.0/) applies to the data made available in this article, unless otherwise stated. 


\section{Background}

Schizophrenia (SZ) is a severe mental disorder with a lifetime prevalence of approximately $1 \%$. Genetic factors are an important part of the aetiology and pathogenesis of SZ [1]. In recent years, in genome-wide association studies, a single nucleotide polymorphism (SNP), rs12807809, in the NRGN gene was reportedly associated with SZ [2], and the $\mathrm{T}$ allele conferred a high risk for SZ. An association of rs12807809 with SZ was also found in 1005 SZ patients and 1069 controls in a South Indian population; further analysis found a moderate association of rs12807809 with flat affect and hallucinations [3]. In addition, an expression quantitative trait loci (eQTL) analysis provided evidence for an increased risk for SZ with rs12807809 at a molecular level [4]. Over the years, rs12807809 has been extensively studied; these studies have suggested that rs12807809 is associated with structural and functional abnormalities in the brain and symptom severity in SZ patients [5-10].

The NRGN gene is the human homologue of the rat $\mathrm{RC} 3 / \mathrm{Ng}$ gene, which is localised to chromosome 11q24.2, spans approximately $12 \mathrm{~kb}$ and contains 4 exons and 3 introns [11]. A 78-amino-acid protein is encoded by exon 1 and exon 2. Exon 3 and exon 4 comprise untranslated sequences. Moreover, intron 1 contains a thyroid hormone-responsive element [12]. NRGN encodes a postsynaptic protein kinase $\mathrm{C}$ (PKC) substrate, which is expressed in the soma and dendrites of neurons in the hippocampus and striatum and in the cerebral cortex [1316]. This protein is called neurogranin $(\mathrm{Ng})$, given that its immunoreactivity is correlated with granule-like structures in hippocampal pyramidal cells in electron micrographs [17]. The phosphorylation of $\mathrm{Ng}$ by $\mathrm{PKC}$ is activated by $\mathrm{Ca}^{2}$ ${ }^{+}$, phospholipids, and diacylglycerol. In the absence of $\mathrm{Ca}^{2+}$, $\mathrm{Ng}$ binds to calmodulin (CaM), which also regulates neuromodulin. Moreover, the phosphorylation of $\mathrm{Ng}$ by $\mathrm{PKC}$ is inhibited by CaM [18]. In the presence of $\mathrm{Ca}^{2+}$, the affinity of $\mathrm{Ng}$ binding to $\mathrm{CaM}$ is decreased. $\mathrm{Ng}$ is considered to regulate many postsynaptic signal transduction pathways due to the role of $\mathrm{Ng}$ in regulating $\mathrm{Ca}^{2+}$ and $\mathrm{CaM}$. $\mathrm{Ng}$ knockout mice showed performance deficits in a spatial learning paradigm. In addition, Ng knockout mice exhibited changes in synaptic plasticity, which suggests the involvement of $\mathrm{Ng}$ in spatial learning and synaptic plasticity [19]. Hippocampal dysfunction has also been associated with rs12807809. In a previous study with 112 healthy volunteers, a relative reduction in hippocampal activation was observed in rs12807809 TT homozygotes compared with that in C-carriers during the acquisition phase of a contextual fear paradigm [20]. The hippocampus is a major component of the brain and plays important roles in learning and memory. Additionally, the hippocampus is the beginning and end of the Papez circuit [21] and an important component of the limbic system.
Hippocampal dysfunction in SZ has been reported in previous magnetic resonance imaging (MRI) studies. Structural studies of SZ have shown abnormalities in hippocampal volume and shape [22-24]. Structural abnormalities in the hippocampal formation have also been reported in populations at a high risk for psychosis [25, 26], implicating genetic susceptibility in hippocampal structural alterations in SZ. Additionally, resting-state functional MRI (fMRI) studies have reported increased hippocampal activation in individuals with SZ [27, 28].

To our knowledge, the association between SNP rs12807809 and hippocampal function in patients with SZ has not been studied. Functional connectivity (FC) is the temporal dependency between spatially remote neurophysiological events [29]. FC can describe the correlation between the time series of anatomically separate brain regions, reflecting the level of functional communication between different regions [29]. In the present study, we investigated the association between rs12807809 and resting-state hippocampal FC in adolescents and adults with SZ and healthy controls (HCs). We hypothesised that rs12807809 would be associated with abnormal hippocampal FC in SZ.

\section{Method}

\section{Participants}

We recruited 59 patients with $\mathrm{SZ}$ (mean age $21.70 \pm \mathrm{SD}$ 9.16 years, range $11-51$ years, $73 \%$ female) from the outpatient clinic at the Department of Psychiatry, the First Hospital of China Medical University, and the Mental Health Center of Shenyang. SZ was diagnosed by 2 associate professors using the Diagnostic and Statistical Manual of Mental Disorders-IV (DSM-IV) criteria. Scores on the Brief Psychiatric Rating Scale (BPRS) were obtained from each participant except for one who did not complete these evaluations. In addition, $99 \mathrm{HC}$ participants (mean age $23.87 \pm$ SD 5.66 years, range $11-50$ years, $58 \%$ female) without any history of mental illness or a family history of psychosis were recruited from the surrounding communities. Most participants were Han Chinese $(n=137,87 \%$ of total participants; $n=53,90 \%$ of patients with SZ; $n=84,85 \%$ of $\mathrm{HCs}$ ), and the ethnicity of the other participants was distributed as follows: Manchu ( $n=13,8 \%$ of total participants; $n=4,7 \%$ of patients with SZ; $n=9,9 \%$ of $\mathrm{HCs}$ ), the Hui nationality ( $n=3,2 \%$ of total participants; $n=3,3 \%$ of HCs), the Mongol nationality ( $n=3,2 \%$ of total participants; $n=1$, $2 \%$ of patients with SZ; $n=2,2 \%$ of $\mathrm{HCs})$, the Korean nationality $(n=1,1 \%$ of total participants; $n=1,2 \%$ of patients with SZ) and the Zhuang nationality $(n=1,1 \%$ of total participants; $n=1,1 \%$ of HCs). The exclusion criteria for both groups were as follows: a history of major physical diseases, particularly those that may be related to changes in cerebral tissue, such as diabetes, 
hypertension, or metastatic tumours; unstable physical conditions, such as severe asthma; a history of nervous system diseases, including major head injury (loss of consciousness lasting more than five minutes), cerebrovascular disease, epilepsy, brain tumours and neurodegenerative diseases; mental retardation autism or extensive developmental disorder; claustrophobia; and strong magnetic objects in the body. All participants signed informed consent after having a sufficient understanding of the study as approved by the Institutional Review Board of China Medical University.

\section{Genotyping}

Genomic DNA was extracted from the venous blood of each participant. The genotypes of rs12807809 were confirmed by the Sanger sequencing technique. Large studies $[3,8,30]$ found a low frequency of the CC genotype (less than $10 \%$ in both patients with SZ and HCs). In our study, the frequency of the CC genotype was $1.7 \%$ in patients with SZ and $12.1 \%$ in HCs. Furthermore, compared to individuals with the $\mathrm{CC}$ or $\mathrm{CT}$ genotypes, the TT genotype may have a higher risk of SZ. Thus, we pooled participants carrying at least one $\mathrm{C}$ allele and compared them with $\mathrm{T}$-allele homozygotes. The participants were further divided into two groups as follows: the C-carrier group ( $29 \mathrm{SZ}, 46 \mathrm{HCs}$; mean age $=23.95 \pm 6.73$ years, $71 \%$ female) and the TT group homozygous for the risk T allele $(30 \mathrm{SZ}, 53 \mathrm{HCs}$; mean age $=22.25 \pm 7.58$ years, $57 \%$ female). The genotype frequencies were in accord with Hardy-Weinberg equilibrium (SZ: $X^{2}=3.732, P=0.053$; HC: $X^{2}=2.893, P=0.089$ ) (Additional file 1: Table S1). The minor allele frequencies were $25 \%$.

\section{Image acquisition and processing}

MRI data were collected by utilising a Signa 3.0 T MRI scanner. Head motion was reduced using foam pads. The participants were required to close their eyes and relax but remain awake. The scanning parameters of the resting-state fMRI were as follows: repetition time $=2000$ $\mathrm{ms}$, echo time $=30 \mathrm{~ms}$, flip angle $=90^{\circ}$, field of view $=24$ $\mathrm{cm} \times 24 \mathrm{~cm}$, matrix $=64 \times 64.35$ axial slices were acquired with a slice thickness of $3 \mathrm{~mm}$ and no gap.

Resting-state fMRI data were preprocessed using the Data Processing Assistant for Resting-State fMRI (DPARSF 2.3, Advanced edition) toolbox [31]. After removing the first ten time points, the following steps were performed: slice timing correction, head motion correction (head motion parameters were calculated by evaluating displacement in each flat and the angular turn around each axis for each volume. The participants were excluded if head motion had a displacement of more than $3 \mathrm{~mm}$ in one or more flats or a $3^{\circ}$ turn around one or more axes between each image. We excluded two patients with SZ and two HCs because of excessive head motion). Spatial normalisation was carried out by employing a standard EPI template from the Montreal Neurological Institute (MNI). The data were resampled to a voxel size of $3 \times 3 \times 3 \mathrm{~mm}^{3}$. A 6 - $\mathrm{mm}$ full-width at half-maximum (FWHM) Gaussian filter was used for the spatial smoothing. Then, further preprocessing steps including linear detrending of physiological noise drift, low-frequency filtering $(0.01-0.08 \mathrm{~Hz})$ and nuisance covariates regression were performed. The nuisance covariates regression eliminated head motion parameters, white matter signal, cerebrospinal fluid signal and global mean signal.

\section{FC analysis}

We defined the bilateral hippocampus as the region of interest (ROI) by using the automated anatomical labelling (AAL) atlas [32] included in REST (REST, V1.8), which was resampled to $3 \times 3 \times 3 \mathrm{~mm}^{3}$. For each participant, we computed the average time course for the hippocampal ROI at the voxel level. Voxel-wise correlation analyses between the hippocampal ROI and other cerebral regions were carried out to generate the FC map. The correlation coefficient map was converted into a $\mathrm{z}$ map by Fisher's r-to-z transformation to improve normality.

\section{Statistical analysis}

We used the chi-square test, independent sample t-test and analysis of variance to examine differences in sex, age, and illness duration among the 4 groups. All statistical analyses were performed using SPSS 22.0 software (SPSS Inc.). All statistical thresholds were set at $P$ value $<0.05$.

The main effect of diagnosis, genotype, and their interaction was assessed using a two-way analysis of covariance in Statistical Parametric Mapping 8 (SPM8) with diagnostic group (SZ and $\mathrm{HC}$ ) and genotype group (CC/ $\mathrm{CT}$ and TT) as the between-subject factors and sex and age as covariates. Statistical significance was defined as a corrected $P<0.05$, with uncorrected thresholds of $P<$ 0.001 and cluster size $>22$ voxels as determined by Gaussian random field (GRF). The FC values of significant regions of interaction were extracted, and the Bonferroni correction was used for post hoc comparisons with a $P<0.05$ considered the threshold for significance. Correlational analyses were performed to determine the correlation of FC values between regions showing significant differences with the BPRS scores in the two diagnostic groups.

\section{Result}

\section{Participant characteristics}

There was no significant difference in age $\left(\mathrm{F}_{3,154}=0.137\right.$, $p=0.712$ ) between the diagnostic and genotype groups. 
Table 1 Demographics and clinical data of participants

\begin{tabular}{|c|c|c|c|c|c|c|}
\hline & \multicolumn{2}{|l|}{$\mathrm{SZ}(n=59)$} & \multicolumn{2}{|l|}{$\mathrm{HC}(n=99)$} & & \multirow[t]{2}{*}{$P$} \\
\hline & $\mathrm{CC} / \mathrm{CT}(n=29)$ & $\Pi(n=30)$ & $\mathrm{CC} / \mathrm{CT}(n=46)$ & $\Pi(n=53)$ & & \\
\hline Age (years), mean $\pm S D$ & $22.86 \pm 8.99$ & $20.57 \pm 9.34$ & $24.63 \pm 4.80$ & $23.21 \pm 6.28$ & $F=0.137$ & 0.712 \\
\hline \multirow[t]{4}{*}{ Gender (male/female) } & $6 / 23$ & $10 / 20$ & $16 / 30$ & $26 / 27$ & $x^{2}=3.727$ & 0.054 (SZ vs. HC) \\
\hline & & & & & $x^{2}=3.343$ & 0.067 (CC/CT vs. TT) \\
\hline & & & & & $x^{2}=2.054$ & 0.152 (HC:CC/CT vs. TT) \\
\hline & & & & & $x^{2}=1.193$ & 0.275 (SZ:CC/CT vs. T) \\
\hline Duration of illness (months), mean \pm SD ${ }^{a}$ & $19.61 \pm 27.94$ & $22.38 \pm 39.40$ & N/A & N/A & $\mathrm{t}=-0.286$ & 0.776 \\
\hline Medication (yes/no) & $25 / 4$ & $25 / 5$ & N/A & N/A & & \\
\hline BPRS score, mean $\pm S D^{b}$ & $32.69 \pm 13.14$ & $33.21 \pm 9.66$ & $18.42 \pm 0.95$ & $18.2 \pm 0.64$ & & \\
\hline
\end{tabular}

SZ Schizophrenia, HC Healthy controls, SD Standard Deviation, BPRS Brief Psychiatric Rating Scale

information is missing for 8 patients. ${ }^{b}$ information is missing for 1 patient

Similarly, there were no significant sex differences between SZ and $\mathrm{HC}\left(\mathrm{x}^{2}=3.727, p=0.054\right)$, between the genotype groups $\left(x^{2}=3.343, p=0.067\right)$, or between the genotype groups within each diagnostic group $\left(\chi^{2}=2.054\right.$, $p=0.152$ in the HC group and $\chi^{2}=1.193, p=0.275$ in the $\mathrm{SZ}$ group). Within the SZ group, no significant difference was found in the duration of illness between the genotype groups $(\mathrm{t}=-0.286, p=0.776)$ (Table 1$)$.

\section{FC analyses}

The main effect of diagnosis, the main effect of genotype and the diagnosis by genotype interaction on FC are listed in Table 2.

Significant main effects of diagnosis were discovered in FC between the hippocampus and bilateral fusiform gyrus, bilateral lingual gyrus, left inferior temporal gyrus, left caudate nucleus, bilateral thalamus and bilateral anterior cingulate gyri (Fig. 1). Compared to HCs, patients with SZ showed significantly increased FC between the hippocampus and bilateral fusiform gyrus, bilateral lingual gyrus, and left inferior temporal gyrus. However, compared to HCs, patients with SZ showed significantly decreased FC between the hippocampus and left caudate nucleus, bilateral thalamus and bilateral anterior cingulate gyri. There was no significant main effect of genotype.

A significant diagnosis by genotype interaction was observed in FC between the hippocampus and bilateral middle cingulate gyri and left anterior cingulate gyrus (Fig. 2). Among patients with SZ, the TT group had significantly lower FC values than the $\mathrm{C}$-carrier group. Within-genotype comparisons showed that TT homozygotes with SZ had a significantly lower FC than HCs who were TT homozygotes.

Correlation analyses between FC and BPRS scores showed no significant associations in the SZ group.

\section{Discussion}

Our study yielded several significant results. First, significantly increased FC between the hippocampus and inferior temporal gyrus, lingual gyrus and fusiform gyrus was observed in patients with SZ compared to HCs. Second, FC was significantly decreased between the hippocampus and caudate nucleus, thalamus and anterior cingulate gyrus in the SZ group compared to the HC group. Third, the lowest FC between the hippocampus and anterior cingulate cortex was found in TT homozygotes with SZ compared to C-carriers with SZ and HC TT homozygotes. These results suggest that the rs12807809 TT genotype in

Table 2 Clusters exhibiting the influence of groups and genotypes on FC

\begin{tabular}{|c|c|c|c|c|c|}
\hline \multirow[t]{2}{*}{ Brain area } & \multirow{2}{*}{$\begin{array}{l}\text { Cluster } \\
\text { size }\end{array}$} & \multicolumn{3}{|c|}{ Peak MNI coordinates } & \multirow{2}{*}{$\begin{array}{l}\text { Peak } \\
\text { F } \\
\text { value }\end{array}$} \\
\hline & & $\bar{x}$ & Y & Z & \\
\hline \multicolumn{6}{|l|}{ Main effect of diagnostic groups } \\
\hline A. Left fusiform gyrus/left lingual gyrus/left inferior temporal gyrus & 782 & -42 & -51 & -12 & 30.30 \\
\hline B. Right lingual gyrus/right fusiform gyrus & 76 & 21 & -57 & -3 & 17.81 \\
\hline C. Left caudate nucleus & 38 & -9 & 9 & 3 & 26.41 \\
\hline D. Left thalamus/right thalamus & 53 & 6 & -6 & 0 & 24.48 \\
\hline E. Left anterior cingulate gyrus/right anterior cingulate gyrus & 70 & 0 & 21 & 21 & 16.64 \\
\hline \multicolumn{6}{|l|}{ Diagnostic groups $\times$ genotype interaction } \\
\hline Left anterior cingulate gyrus/left middle cingulate gyrus/right middle cingulate gyrus & 101 & 0 & 6 & 33 & 23.21 \\
\hline
\end{tabular}

These findings correspond to a corrected $P<0.05$ by GRF correction. $B A$ Brodmann's area. Cluster size is in $\mathrm{mm}^{3}$ 




b

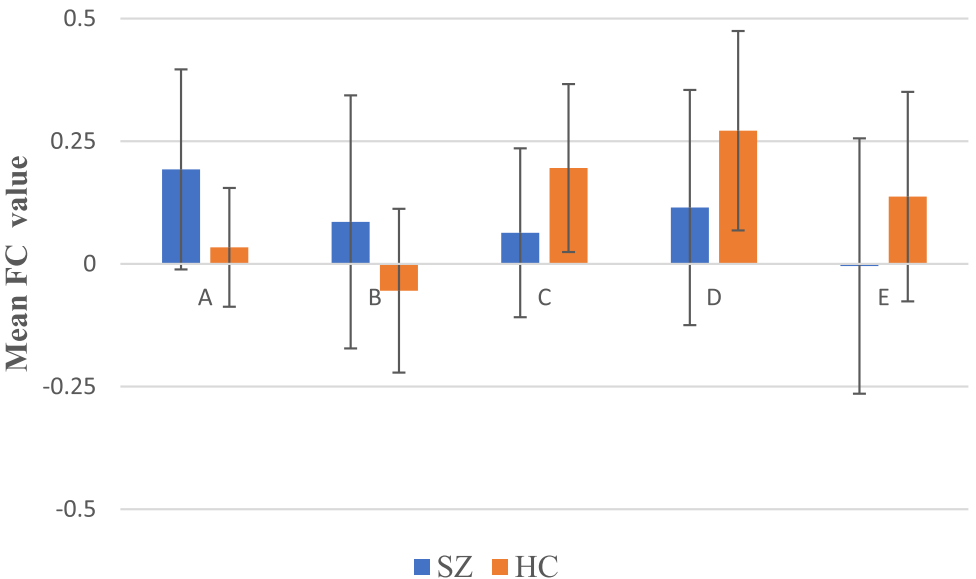

Fig. 1 The main effect of diagnostic group. (a) Regions (white box) with main effect of diagnostic group include (a) left fusiform gyrus, left lingual gyrus, left inferior temporal gyrus, (b) right lingual gyrus, right fusiform gyrus, (c) left caudate nucleus, (d) left thalamus, right thalamus, (e) left anterior cingulate gyrus, right anterior cingulate gyrus (cluster-level threshold of $p<0.05$ after GRF correction and cluster size $=35$ ). The coloured bar represents the range of $F$ values. (b) Shown here are the FC values (mean \pm standard deviation) extracted from regions with main effect of diagnostic group. The Y-axis represents FC values. The X-axis represents regions with main effect of diagnostic group

the NRGN gene is associated with abnormal hippocampal $\mathrm{FC}$ at rest in SZ.

Compared to HCs, SZ patients exhibited increased FC between the hippocampus and fusiform gyrus, lingual gyrus, and inferior temporal gyrus. The increased FC between the hippocampus and lingual gyrus is consistent with previously reported changes in a longitudinal resting-state fMRI study [33]. Kraguljac et al. reported significantly increased left posterior hippocampal FC with the lingual gyrus in 34 drug-naïve patients with SZ compared to $34 \mathrm{HCs}$. The lingual gyrus is involved in visual processing and encoding visual memories [34]. The disconnect between the lingual gyrus and hippocampus in patients with SZ may be associated with an 



Fig. 2 Diagnosis by genotype interaction. (a) Significant regions of diagnosis by genotype interaction from two-way ANCOVA include the bilateral middle cingulate gyri and left anterior cingulate gyrus $(P<0.05$ by GRF correction and 22 voxels minimum). The coloured bar represents the range of $F$ values. (b) Shown here are the mean ( \pm standard deviation) FC values extracted from significant regions of the diagnosis by genotype interaction. Post hoc two-sample t-tests show that $\Pi$ homozygotes with SZ have significantly lower $\mathrm{FC}$ values than do healthy $\Pi \pi$ homozygotes and C-carriers with SZ. ${ }^{*}, P<0.05$

impairment of visual memory. Moreover, in the present study, increased FC was observed between the hippocampus and inferior temporal gyrus and fusiform gyrus. Few studies have reported abnormal FC between the hippocampus and inferior temporal gyrus and fusiform gyrus in patients with SZ. The fusiform gyrus participates in the within-category identification, word recognition, face and body recognition, and processing of colour information [34]. The main function of the inferior temporal gyrus is receiving information and object 
recognition [35]. The inferior temporal gyrus, fusiform gyrus and hippocampus work together to produce an understanding of the physical world. Abnormal FC between the hippocampus and fusiform gyrus and inferior temporal gyrus may be associated with disorientation in patients with SZ.

Decreased FC between the hippocampus and caudate nucleus, thalamus and anterior cingulate gyrus was found in the SZ group compared to that in the HC group. This decreased FC is consistent with some [36, 37] but not all [33] previous fMRI studies, indicating that hippocampal FC changes in patients with SZ without regard to genetic influences. Samudra et al. reported reduced hippocampal FC with the thalamus in 88 patients with psychosis compared to that in 65 HCs. Furthermore, anterior and posterior hippocampal ROI analyses showed reduced connectivity with the anterior cingulate cortex and thalamus. In another longitudinal study of 68 patients with first-episode SZ and $62 \mathrm{HCs}$, decreased FC with the cingulate cortex in the bilateral hippocampus network was found in patients compared with that in healthy controls at both baseline and follow-up. However, Kraguljac et al. reported significantly increased left anterior hippocampal FC with the caudate nucleus and anterior cingulate cortex in 34 drug-naïve patients with SZ compared to that in 34 HCs. The differences in the results among these studies may be associated the effect of antipsychotics. The Papez circuit was proposed by James Papez in 1937 [38] and involves various areas of the limbic system, such as the hippocampus and cingulate gyrus and the anterior thalamic nucleus [21]. The Papez circuit, which is believed to be involved in memory, specifically spatial and episodic memory $[39,40]$, is damaged in patients with SZ. Although our previous studies reported dysfunction of the Papez circuit in major depressive disorder [41], few studies have shown the function of the Papez circuit in SZ thus far. Our findings suggest that the Papez circuit may be involved in the pathophysiology of SZ. However, more effort is necessary to elucidate the mechanisms by which Papez circuit deficits are involved in the pathophysiology of SZ.

Although no difference was found in hippocampal FC between genotype groups, the lowest FC between the hippocampus and anterior cingulate gyrus was observed in TT homozygotes with SZ compared to that in C-carriers with SZ and HC TT homozygotes. In SZ studies, Ohi and colleagues also reported reduced grey matter volume in the left anterior cingulate cortex in TT homozygotes compared to that in C-carriers [5]. In addition, an fMRI study including 94 healthy participants showed stronger activation in the anterior cingulate cortex in TT homozygotes than in C-carriers during episodic memory encoding [42]. NRGN has the potential to influence hippocampal plasticity, spatial learning and long-term potentiation. Moreover, the anterior cingulate cortex participates in both cognitive and emotional processing [43, 44]; this structure is mainly involved in the modulation of attention or executive functions, error detection, working memory, episodic memory, the regulation of emotional responses, and in the salience of emotional and motivational information [43-47]. Both the anterior cingulate cortex and hippocampus are parts of the Papez circuit and are involved in episodic memory encoding. Therefore, our finding of abnormal FC between the hippocampus and anterior cingulate cortex suggests that the $\mathrm{T}$ risk allele may influence the Papez circuit deficits observed in SZ.

The current study has some limitations. First, the sample size was not large enough; thus, a false positive result may have been obtained. Therefore, a study with a larger sample should be conducted to further assess the effect of genes. Second, other SNPs in this gene or other genes involved in SZ were not included in our study. Third, we did not consider the effect of antipsychotics and the course of the disease in patients with SZ. Further studies should be conducted in first-episode, drug-naïve patients with SZ.

\section{Conclusion}

In conclusion, for the first time, the present study verifies that the NRGN rs12807809 TT genotype may be associated with Papez circuit dysfunction in patients with SZ. This finding may implicate NRGN variations and Papez circuit dysfunction in SZ pathophysiology.

\section{Additional file}

Additional file 1: Supplementary materials. (DOCX 14 kb)

\section{Abbreviations}

BPRS: Brief Psychiatric Rating Scale; CaM: Calmodulin; FC: Functional connectivity; HC: Healthy control; Ng: Neurogranin; PKC: Protein kinase C; ROI: Region of interest; SZ: Schizophrenia

\section{Acknowledgements}

The author wishes to thank all teachers, classmates and colleagues in the Department of Psychiatry and Radiology of The First Affiliated Hospital of China Medical University, as well as all participants.

\section{Funding}

The authors were supported by research grants from the National Natural Science Foundation of China Funding support: National Natural Science Foundation of China (81571311 to Y.T., 81571331 to F.W.), National Science Fund for Distinguished Young Scholars (81725005 to F.W.), National Key Research and Development Program (2016YFC1306900 to Y.T., 2016 YFC0904300 to F.W.), National High Tech Development Plan (863) (2015AA020513 to F.W.), Liaoning Science and Technology Project (2015225018 to Y.T.), Liaoning Education Foundation (Pandeng Scholar to F.W.). The funders had no influence on the research design, data collection, data analysis, interpretation of results, writing the manuscript or submitting for publication. 


\section{Availability of data and materials}

The datasets used and/or analysed during the current study are available from the corresponding author on reasonable request.

\section{Authors' contributions}

$Y Z 1, X G$, FW and YT designed the study. YZ1, ZY, LC, JY, and PW collected participants. $Y Z 1, X G, Z Y, L C, Y Z 2, X J$, and SW performed the analysis. YZ1 drafted the manuscript. All authors read, contributed to and approved the final manuscript.

\section{Ethics approval and consent to participate}

The study was approved by the Medical Science Research Ethics Committee of the First Affiliated Hospital of China Medical University (approval reference number[2012]25-1). All participants gave written informed consent before taking part in this study. Patients signed informed consent with the consent of their guardian, and all minor participants and their parents signed informed consent.

\section{Consent for publication}

Authors have obtained consent to publish from the participants to report patient data.

\section{Competing interests}

The authors declare that they have no competing interests.

\section{Publisher's Note}

Springer Nature remains neutral with regard to jurisdictional claims in published maps and institutional affiliations.

\section{Author details \\ ${ }^{1}$ Department of Psychiatry, The First Affiliated Hospital of China Medical University, Shenyang, Liaoning 110001, People's Republic of China. ${ }^{2}$ State Key Laboratory of Genetic Engineering and MOE Key Laboratory of Contemporary Anthropology, School of Life Sciences, Fudan University, Shanghai 200433, People's Republic of China. ${ }^{3}$ Department of Radiology, The First Affiliated Hospital of China Medical University, Shenyang, Liaoning 110001, People's Republic of China. ${ }^{4}$ Department of Psychiatry and Gerontology, The First Affiliated Hospital of China Medical University, 155 Nanjing North Street, He ping District, Shenyang, Liaoning 110001, People's Republic of China. ${ }^{5}$ Brain Function Research Section and Department of Psychiatry and Radiology, The First Affiliated Hospital of China Medical University, 155 Nanjing North Street, He ping District, Shenyang, Liaoning 110001, People's Republic of China.}

\section{Received: 10 July 2018 Accepted: 24 March 2019}

\section{Published online: 05 April 2019}

\section{References}

1. Sullivan PF, Kendler KS, Neale MC. Schizophrenia as a complex trait: evidence from a meta-analysis of twin studies. Arch Gen Psychiatry. 2003; 60(12):1187-92.

2. Stefansson H, Ophoff RA, Steinberg S, Andreassen OA, Cichon S, Rujescu D, Werge T, Pietiläinen $\mathrm{OPH}$, Mors $\mathrm{O}$, Mortensen $\mathrm{PB}$, et al. Common variants conferring risk of schizophrenia. Nature. 2009.

3. Sudesh R, Priyadarshini T, Preeti R, John S, Thara R, Mowry B, Munirajan AK Minor allele C of rs12807809 polymorphism in NRGN contributes to the severity of psychosis in patients with schizophrenia in south Indian population. Neurosci Lett. 2017;649:107-11.

4. Blauwendraat C, Francescatto M, Gibbs JR, Jansen IE, Simón-Sánchez J, Hernandez DG, Dillman AA, Singleton AB, Cookson MR, Rizzu P, et al. Comprehensive promoter level expression quantitative trait loci analysis of the human frontal lobe. Genome Medicine. 2016:8(1):65.

5. Ohi K, Hashimoto R, Yasuda Y, Nemoto K, Ohnishi T, Fukumoto M, Yamamori H, Umeda-Yano S, Okada T, Iwase M, et al. Impact of the genome wide supported NRGN gene on anterior cingulate morphology in schizophrenia. PLoS One. 2012;7(1):e29780.

6. Rose EJ, Morris DW, Fahey C, Robertson IH, Greene C, O'Doherty J, Newell FN, Garavan H, McGrath J, Bokde A, et al. The effect of the neurogranin schizophrenia risk variant rs12807809 on brain structure and function. Twin Res Hum Genet. 2012;15(3):296-303.
7. Liu C, Thong JYJ, Qiu A, Sum MY, Kuswanto CN, Tuan TA, Donohoe G, Sitoh YY, Sim K. Effects of the Neurogranin variant rs12807809 on Thalamocortical morphology in schizophrenia. PLoS One. 2013;8(12):e85603.

8. Ohi K, Hashimoto R, Yasuda Y, Fukumoto M, Yamamori H, Umeda-Yano S, Fujimoto $M$, Iwase $M$, Kazui $H$, Takeda M. Influence of the NRGN gene on intellectual ability in schizophrenia. J Hum Genet. 2013;58(10):700-5.

9. Walton E, Geisler D, Hass J, Liu J, Turner J, Yendiki A, Smolka MN, Ho BC, Manoach DS, Gollub RL, et al. The impact of genome-wide supported schizophrenia risk variants in the neurogranin gene on brain structure and function. PLoS One. 2013;8(10):e76815.

10. Su L, Long J, Pan R, Xie X, Mao X, Zhou Y, Chen Q, Wei B. Influence of NRGN rs12807809 polymorphism on symptom severity in individuals with schizophrenia in the Han population but not the Zhuang population of South China. Acta neuropsychiatrica. 2015;27(4):221-7.

11. Martinez de Arrieta C, Perez Jurado L, Bernal J, Coloma A: Structure, organization, and chromosomal mapping of the human neurogranin gene (NRGN). Genomics 1997, 41(2):243-249.

12. Martinez de Arrieta C, Morte B, Coloma A, Bernal J: The human RC3 gene homolog, NRGN contains a thyroid hormone-responsive element located in the first intron. Endocrinology 1999, 140(1):335-343.

13. Represa A, Deloulme JC, Sensenbrenner M, Ben-Ari Y, Baudier J. Neurogranin: immunocytochemical localization of a brain-specific protein kinase C substrate. J Neurosci. 1990;10(12):3782-92.

14. Watson JB, Sutcliffe JG, Fisher RS. Localization of the protein kinase $C$ phosphorylation/calmodulin-binding substrate RC3 in dendritic spines of neostriatal neurons. Proc Natl Acad Sci U S A. 1992;89(18):8581-5.

15. Watson JB, Szijan I, Coulter PM 2nd. Localization of RC3 (neurogranin) in rat brain subcellular fractions. Brain Res Mol Brain Res. 1994;27(2):323-8.

16. Neuner-Jehle M, Denizot JP, Mallet J. Neurogranin is locally concentrated in rat cortical and hippocampal neurons. Brain Res. 1996;733(1):149-54.

17. Baudier J, Deloulme JC, Van Dorsselaer A, Black D, Matthes HW. Purification and characterization of a brain-specific protein kinase C substrate, neurogranin (p17). Identification of a consensus amino acid sequence between neurogranin and neuromodulin (GAP43) that corresponds to the protein kinase $C$ phosphorylation site and the calmodulin-binding domain. J Biol Chem. 1991;266(1):229-37.

18. Huang KP, Huang FL, Chen HC. Characterization of a $7.5-\mathrm{kD}$ a protein kinase C substrate (RC3 protein, neurogranin) from rat brain. Arch Biochem Biophys. 1993;305(2):570-80.

19. Pak JH, Huang FL, Li J, Balschun D, Reymann KG, Chiang C, Westphal H, Huang KP. Involvement of neurogranin in the modulation of calcium/calmodulindependent protein kinase II, synaptic plasticity, and spatial learning: a study with knockout mice. Proc Natl Acad Sci U S A. 2000;97(21):11232-7.

20. Pohlack ST, Nees F, Ruttorf M, Witt SH, Nieratschker V, Rietschel M, Flor H. Risk variant for schizophrenia in the neurogranin gene impacts on hippocampus activation during contextual fear conditioning. Mol Psychiatry. 2011;16(11):1072-3.

21. Shah A, Jhawar SS, Goel A. Analysis of the anatomy of the Papez circuit and adjoining limbic system by fiber dissection techniques. Journal of clinical neuroscience : official journal of the Neurosurgical Society of Australasia. 2012;19(2):289-98.

22. Ota M, Sato N, Hidese S, Teraishi T, Maikusa N, Matsuda H, Hattori K, Kunugi H. Structural differences in hippocampal subfields among schizophrenia patients, major depressive disorder patients, and healthy subjects. Psychiatry Res. 2017:259:54-9.

23. Kalmady SV, Shivakumar V, Arasappa R, Subramaniam A, Gautham S, Venkatasubramanian G, Gangadhar BN. Clinical correlates of hippocampus volume and shape in antipsychotic-naive schizophrenia. Psychiatry Res. 2017:263:93-102.

24. Chang M, Womer FY, Bai C, Zhou Q, Wei S, Jiang X, Geng H, Zhou Y, Tang $Y$, Wang F. Voxel-based morphometry in individuals at genetic high risk for schizophrenia and patients with schizophrenia during their first episode of psychosis. PLoS One. 2016;11(10):e0163749.

25. Dean DJ, Orr JM, Bernard JA, Gupta T, Pelletier-Baldelli A, Carol EE, Mittal VA. Hippocampal shape abnormalities predict symptom progression in neuroleptic-free youth at ultrahigh risk for psychosis. Schizophr Bull. 2016: 42(1):161-9.

26. Bois C, Levita L, Ripp I, Owens DC, Johnstone EC, Whalley HC, Lawrie SM. Hippocampal, amygdala and nucleus accumbens volume in first-episode schizophrenia patients and individuals at high familial risk: a cross-sectional comparison. Schizophr Res. 2015;165(1):45-51. 
27. McHugo M, Rogers BP, Talati P, Woodward ND, Heckers S. Increased amplitude of low frequency fluctuations but Normal hippocampal-default mode network connectivity in schizophrenia. Frontiers in psychiatry. 2015;6:92.

28. Tregellas JR, Smucny J, Harris JG, Olincy A, Maharajh K, Kronberg E, Eichman LC, Lyons E, Freedman R. Intrinsic hippocampal activity as a biomarker for cognition and symptoms in schizophrenia. Am J Psychiatry. 2014;171(5): 549-56.

29. van den Heuvel MP, Hulshoff Pol HE. Exploring the brain network: a review on resting-state fMRI functional connectivity. Eur Neuropsychopharmacol. 2010;20(8):519-34.

30. Wen Z, Chen J, Khan RA, Wang M, Song Z, Li Z, Shen J, Li W, Shi Y. Polymorphisms in NRGN are associated with schizophrenia, major depressive disorder and bipolar disorder in the Han Chinese population. J Affect Disord. 2016;194:180-7.

31. Chao-Gan Y, Yu-Feng Z. DPARSF: a MATLAB toolbox for "pipeline" data analysis of resting-state fMRI. Front Syst Neurosci. 2010;4(13):13.

32. Tzourio-Mazoyer N, Landeau B, Papathanassiou D, Crivello F, Etard O, Delcroix N, Mazoyer B, Joliot M. Automated anatomical labeling of activations in SPM using a macroscopic anatomical parcellation of the MNI MRI single-subject brain. Neurolmage. 2002;15(1):273-89.

33. Kraguljac NV, White DM, Hadley N, Hadley JA, Ver Hoef L, Davis E, Lahti AC. Aberrant hippocampal connectivity in Unmedicated patients with schizophrenia and effects of antipsychotic medication: a longitudinal resting state functional MRI study. Schizophr Bull. 2016:42(4):1046-55.

34. Bogousslavsky J, Miklossy J, Deruaz JP, Assal G, Regli F. Lingual and fusiform gyri in visual processing: a clinico-pathologic study of superior altitudinal hemianopia. J Neurol Neurosurg Psychiatry. 1987;50(5):607-14.

35. Denys K, Vanduffel W, Fize D, Nelissen K, Peuskens H, Van Essen D, Orban GA. The processing of visual shape in the cerebral cortex of human and nonhuman primates: a functional magnetic resonance imaging study. J Neurosci. 2004;24(10):2551-65.

36. Samudra N, Ivleva El, Hubbard NA, Rypma B, Sweeney JA, Clementz BA, Keshavan MS, Pearlson GD, Tamminga CA. Alterations in hippocampal connectivity across the psychosis dimension. Psychiatry Res. 2015;233(2): $148-57$

37. Duan HF, Gan JL, Yang JM, Cheng ZX, Gao CY, Shi ZJ, Zhu XQ, Liang XJ, Zhao LM. A longitudinal study on intrinsic connectivity of hippocampus associated with positive symptom in first-episode schizophrenia. Behav Brain Res. 2015;283:78-86.

38. Papez JW: A proposed mechanism of emotion. 1937. The Journal of neuropsychiatry and clinical neurosciences 1995, 7(1):103-112.

39. Nishio Y, Hashimoto M, Ishii K, Mori E. Neuroanatomy of a neurobehavioral disturbance in the left anterior thalamic infarction. J Neurol Neurosurg Psychiatry. 2011:82(11):1195-200.

40. Jankowski MM, Ronnqvist KC, Tsanov M, Vann SD, Wright NF, Erichsen JT, Aggleton JP, O'Mara SM. The anterior thalamus provides a subcortical circuit supporting memory and spatial navigation. Front Syst Neurosci. 2013;7:45.

41. Jiang W, Gong G, Wu F, Kong L, Chen K, Cui W, Ren L, Fan G, Sun W, Ma H, et al. The papez circuit in first-episode, treatment-naive adults with major depressive disorder: combined atlas-based tract-specific quantification analysis and voxel-based analysis. PLoS One. 2015;10(5):e0126673.

42. Krug A, Krach S, Jansen A, Nieratschker V, Witt SH, Shah NJ, Nothen MM Rietschel M, Kircher T. The effect of neurogranin on neural correlates of episodic memory encoding and retrieval. Schizophr Bull. 2013;39(1):141-50.

43. Vogt BA, Finch DM, Olson CR: Functional heterogeneity in cingulate cortex: the anterior executive and posterior evaluative regions. Cerebral cortex (New York, NY: 1991) 1992, 2(6):435-443.

44. Devinsky $\mathrm{O}$, Morrell MJ, Vogt BA: Contributions of anterior cingulate cortex to behaviour. Brain 1995, 118 ( Pt 1):279-306.

45. Carter CS, Botvinick MM, Cohen JD. The contribution of the anterior cingulate cortex to executive processes in cognition. Rev Neurosci. 1999; 10(1):49-57.

46. Bush G, Frazier JA, Rauch SL, Seidman $\sqcup$, Whalen PJ, Jenike MA, Rosen BR, Biederman J. Anterior cingulate cortex dysfunction in attention-deficit/ hyperactivity disorder revealed by fMRI and the counting Stroop. Biol Psychiatry. 1999;45(12):1542-52.

47. Kiehl KA, Liddle PF, Hopfinger JB. Error processing and the rostral anterior cingulate: an event-related fMRI study. Psychophysiology. 2000;37(2):216-23.

\section{Ready to submit your research? Choose BMC and benefit from}

- fast, convenient online submission

- thorough peer review by experienced researchers in your field

- rapid publication on acceptance

- support for research data, including large and complex data types

- gold Open Access which fosters wider collaboration and increased citations

- maximum visibility for your research: over $100 \mathrm{M}$ website views per year

At BMC, research is always in progress.

Learn more biomedcentral.com/submissions 